\title{
Virtual Reality for Acute Pain in Outpatient Hysteroscopy: A Randomised Controlled Trial
}

\author{
Nandita Deo ${ }^{1}$, Khalid Khan ${ }^{2}$, Jonathan Mak $^{3}$, John Allotey ${ }^{3}$, Francisco Jose Gonzalez \\ Carreras $^{4}$, Gianpaolo Fusari ${ }^{5}$, and Jonathan Benn ${ }^{6}$ \\ ${ }^{1}$ Whipps Cross University Hospital NHS Trust \\ ${ }^{2}$ University of Granada \\ ${ }^{3}$ Queen Mary University of London \\ ${ }^{4}$ Queen Mary University of London, 58 Turner Street Yvonne Carter Building \\ ${ }^{5}$ Imperial College London and the Royal College of Art \\ ${ }^{6}$ University of Leeds
}

May 5, 2020

\begin{abstract}
Objective: To evaluate the effectiveness of virtual reality as a distraction technique in the management of acute pain and anxiety during outpatient hysteroscopy. Methods: A parallel group, prospective randomised controlled trial was conducted at a UK University Hospital (August to October 2018) (ClinicalTrials.gov Id: NCT03699280). Forty consenting, eligible women were randomised to virtual reality intervention (immersive video content as a distraction method) or standard care during outpatient hysteroscopy. Pain and anxiety outcomes were measured as a numeric rating score (scale of 0-10). Results: Compared to standard care, women with virtual reality intervention experienced less average pain (score 6.0 vs 3.7 , mean difference $2.3,95 \%$ CI $0.61-3.99, \mathrm{p}=0.009$ ) and anxiety (score 5.45 vs 3.3 , mean difference $2.15,95 \%$ CI 0.38-3.92, p=0.02). Conclusion: Virtual Reality was effective in reducing pain and anxiety during outpatient hysteroscopy in a pilot trial. Its wide potential role in ambulatory gynaecologic procedures needs further evaluation.
\end{abstract}

\section{Introduction}

Performance of diagnostic and operative procedures for gynaecological conditions in the consultation room setting, is becoming increasingly commonplace in order to reduce risks of general anaesthetic, decrease health care costs and increase convenience for both patient and provider $^{1}$. Such procedures are usually well tolerated (reference), can be associated with acute pain and anxiety ${ }^{2}{ }^{3},{ }^{45}{ }^{6}$. Pain relief options include sedation, local anaesthetic, analgesics and distraction techniques, though no consistent good quality evidence exists to underpin practice 89101112 .

Virtual reality, a relatively new intervention, has been studied as a distraction technique for nonpharmacological pain relief. Put simply, it is a computer-generated representation of an immersive environment viewed through a headset ${ }^{13}$. The cost, quality and accessibility of virtual reality devices has significantly improved in recent years and offered novel application in the medical field. Virtual reality for managing pain has been studied in paediatrics, dentistry, burns treatment, chronic pain, labour, episiotomy and phobias ${ }^{14-22} 2324$. Although a metaanalysis suggested that VR may have a role in reducing pain scores in acutely painful procedures, it was found to be effective only in needles and burns physical therapy. The studies of VR on pain and anxiety however were limited by clinical and statistical heterogeneity ${ }^{14}$ ${ }^{25}$ Nonpharmacological options of pain relief have not explored the role of virtual reality in reducing pain and 
improving patient experience in outpatient hysteroscopy ${ }^{26}$. To our knowledge, there are no publications studying the effects of Virtual Reality in the management of pain during office gynaecological procedures ${ }^{7}$

We conducted a randomised controlled trial of virtual reality intervention as a distraction technique, versus standard care, in managing acute pain and anxiety during outpatient hysteroscopy.

\section{Methods}

\section{Study design and setting}

The study was a single centre, parallel group, prospective randomised controlled trial conducted at a large University hospital in London UK from August 2018 to October 2018 (Whipps Cross University Hospital). The study was approved by the National Research Ethics Committee, Health Research Authority and registered as a clinical trial. (ClinicalTrials.gov Identifier: NCT03699280). This research did not receive any external funding.

\section{Study participants and eligibility criteria}

Consecutive women scheduled to undergo an outpatient hysteroscopy were invited to participate in the trial. Eligibility criteria included all consenting women $18-70$ years of age with a planned outpatient hysteroscopy. Excluded were any women with hearing or visual impairment, or any known anatomical characteristics that makes performing the office procedure difficult, e.g. cervical conization, amputation.

\section{Recruitment, randomisation and follow up}

After written informed consent, eligible women were randomly allocated using sealed envelopes to either the virtual reality intervention or standard care. Using a secure online system, a randomisation scheme based on permuted block of random block sizes $(2,4)$ and stratified by parity (nulliparous, multiparous) and menopausal status (premenopausal, post- menopausal), created the allocation sequence. Due to the nature of the intervention, blinding of participants, care providers and outcome assessors was not possible, but allocation remained concealed until randomisation.

The intervention group received the virtual reality device with immersive video content for the use during their outpatient hysteroscopy as a distraction method. In the standard care group, women underwent their outpatient hysteroscopy as a routine procedure without offering the virtual reality intervention. Patient follow up was clinically indicated, not arranged for the purpose of the trial.

\section{Outpatient hysteroscopy (standard care)}

All procedures were performed in the office setting using a $3.2 \mathrm{~mm}$ rigid hysteroscope using normal saline as distension medium. A vaginoscopic technique was utilised unless it failed and dilatation was necessary. Depending on the indications and findings of the hysteroscopy, additional procedures like pipelle biopsies, endometrial biopsies using biopsy forceps, polypectomies, Mirena coil insertions or removals were recorded. Intracervical local anaesthetic infiltration was administered where necessary in the form of rescue analgesia.

\section{Virtual reality during hysteroscopy (intervention)}

Immersive and interactive video content was delivered to patients randomised to the virtual reality intervention using an portable, standalone VR headset called Oculus Go with a head mounted display with built in audio drivers. The guided relaxation experience included viewing an 8-minute video called 'Forest of Serenity' commissioned by St. Giles Hospice, developed by Holosphere and narrated by Sir David Attenborough ${ }^{27}$. The immersive video simulated a calming rainforest and a lake setting with animated wildlife, which could be explored by using the headtracker. The video played was one with minimal movement and a familiar voice to achieve maximal desired effect. The video was played for the duration of the procedure and replayed when the procedure exceeded 8 minutes. Patients were allowed to stop viewing the video or remove the headset at their own discretion or in the event of side effects.

\section{Outcomes and measurements}


Primary outcome measurements were worst and average pain, based on numeric rating scores (11-point scale from 0 to 10; 0 representing 'no pain/anxiety' and 10 representing 'worst imaginable pain/anxiety') along with anxiety, recorded pre-procedurally (as 'anticipated' prior to the procedure) and that 'experienced' during the procedure $^{28},{ }^{29,30}$. Data was collected immediately before and after the procedure. Data on the proportion of patients eligible, stratification factors (menopausal status and parity) consented and randomised, reasons for non-participation, and acceptability of the trial and intervention to participants and healthcare providers, were collected. The perception of the clinician performing the procedure and the nursing staff regarding feasibility of using the virtual reality equipment for each patient who had the intervention was assessed through questionnaires. Semi-structured interviews were conducted with women who received the virtual reality intervention within 30 minutes of the procedure and were recorded on a digital voice recorder. The questions focused on the patient's experience of the hysteroscopy and the intervention, pain and anxiety perceived and also any other aspects that they felt were relevant to hospital care. The interviews allowed for all participants to be asked similar questions within a flexible framework. ${ }^{31}$

Interviews continued until no new information was being obtained and theoretical saturation point was reached. ${ }^{32}$

\section{Sample size and statistical analysis}

The target sample size for this trial was 40 (20 per group), based on the weekly number of women attending who could be approached (15) and an estimated $60 \%$ participation rate. There were no prior estimates of standard deviations available for power estimation. All data was entered into a secure database and anonymised using participant codes at the point of data entry.

Statistical analysis was by intention-to-treat including all randomised participants, using $\mathrm{R}$ software Version 3.5.1 (Feather Spray). Continuous data were summarised as mean and standard deviation, and categorical data as counts and percentages. Between-group differences were reported with $95 \%$ confidence intervals (CI) and p-value (using t test to compare normally distributed data). Cohen's d, difference in scores measured on a standard deviation scale, was used to determine effect size with values above 0.7 considered to be large ${ }^{33}$. Linear regression was used to estimate difference in continuous outcomes between groups post-procedure, adjusting for stratification factors (of menopausal status and parity) and baseline score.

\section{Patient and public engagement}

Prior to the study, the development of the research question was informed by patient's priorities and preferences. Staff and patients were involved in the planning of the study and in designing the intervention including the selection of videos for viewing. Patients and public representatives were not involved in the recruitment or the conduct of the study. Interviews and focus group discussions gathering information on the implementation, acceptability and content of the virtual reality videos viewed with clinical staff, was done to get an understanding of factors that might influence participation in a definitive trial.

\section{Results}

\section{Patient recruitment and characteristics}

A total of 53 women were approached for 6 weeks between August 2018 and October 2018. Of these, 8 declined to participate and 5 did not meet eligibility criteria. Finally, forty of 48 (83\%) women agreed to participate and were randomised. (Figure 1). Reasons for exclusion of the 5 patients included 4 patients being over the age of 70, of which one patient had hearing difficulty and 1 patient did not need a hysteroscopy. Eight patients declined to participate of which 2 patients wanted to see the procedure, 2 patients had used virtual reality before for gaming and were queasy, 2 patients were very anxious about the procedure and declined participation, 1 patient couldn't wait for the procedure as there were delays in the clinic and 1 patient had brought her own headphones with an audio track to keep herself distracted. All patients completed the procedure except one having standard care who did not tolerate the procedure and needed to be booked for an outpatient hysteroscopy under general anaesthetic. Data for all 40 patients was considered for statistical analysis. 
Baseline characteristics (Table 1) show that groups were balanced for features including age, parity, menopausal status, previous experience of outpatient hysteroscopy, anticipated pain and anxiety scores, and analgesic intake prior to the procedure. Before the procedure, the mean expected pain and anxiety scores were 6.7 and 5.98 respectively and there were no significant differences in either score between standard care and virtual reality groups. The procedures were performed in a single centre by 4 clinicians of consultant grade and a nurse and a healthcare assistant supported the clinics. Vaginoscopic approach was possible in $90 \%(36 / 40)$ of all the procedures. The mean duration of the procedure was 4.82 minutes (Range 1.5 minutes to 14 minutes). Local anaesthetic infiltration was required for pain management in 15\% (6/40) of the $18 \%(7 / 40)$ of women had cervical stenosis. Eighteen percentage of the patients $(7 / 40)$ had an experience of an outpatient hysteroscopy in the past.

Nausea was experienced by one patient in the virtual reality intervention arm, however she kept the headset on until the end of the procedure; one patient had previous history of claustrophobia and decided to removed the headset when the procedure started as she felt claustrophobic.

\section{Pain and anxiety}

Compared to standard care, the virtual reality intervention had a large effect reducing worst pain with a 2.2 score difference ( $28 \%$ reduction, score 7.85 vs. $5.65,95 \%$ CI $3.79-3.79, \mathrm{p}=0.011$, Cohen's d 0.82 ), average pain with a 2.3 difference ( $38 \%$ reduction, score 6.0 vs. $3.7,95 \%$ CI $0.61-3.99, \mathrm{p}=0.009$, Cohen's d 0.81 ), and anxiety with a 2.15 difference ( $39 \%$ reduction, scores 5.45 vs. $3.3,95 \%$ CI $0.38-3.92, \mathrm{p}=0.024$, Cohen's d 0.73$)^{14}$ (Table 2, Appendix S1).

In order to examine whether the observed effects of virtual reality were robust, multiple regression models were fitted for each pain and anxiety outcome, to estimate the effect of the virtual reality condition, whilst controlling for baseline (expected) pain/anxiety, parity, menopausal condition and cervical stenosis (Table 3; Appendix S1). For worst pain scores, the virtual reality condition accounted for a 2.11-point decrease in experienced pain, compared with the control group $(\mathrm{p}=0.011 ; \mathrm{R} 2=0.24)$, after controlling for baseline scores and covariates. For average pain scores, a 2.28-point decrease in experienced pain was observed $(\mathrm{p}=0.01$; $\mathrm{R} 2=0.24)$ and for anxiety scores, a 2.13-point decrease $(\mathrm{p}=0.024 ; \mathrm{R} 2=0.16)$.

Follow up questionnaire results revealed that all (100\%) of the women who received the virtual reality intervention were happy to have the procedure again in the outpatient setting. Fifteen percent $(6 / 40)$ women receiving standard care expressed their views that they would have liked to have the procedure done under general anaesthetic instead of the outpatient setting.

The gynaecologists performing the procedure reported that the intervention was feasible in $90 \%(18 / 20)$ and thought to be helpful for the particular patient in $85 \%(17 / 20)$ of cases. The staff nurses assisting the procedure reported that the intervention was feasible in $85 \%(17 / 20)$ and thought to be helpful for the particular patient in $85 \%(17 / 20)$ of cases

\section{Patient and staff experience}

Semi-structured interviews were conducted with patients (16 who received virtual reality intervention and 12 patients who had standard care), 2 clinical staff and 3 nursing staff (Appendix S2). Thematic analysis of interview transcripts provided rich insights into patients' experience of the VR intervention. A range of representative quotes from patients (Appendix S2) illustrates the possible mechanisms by which virtual reality immersion was reported to influence the experience of pain and anxiety. Positive experiences included a sense of relaxation that distracted from pain, as a result of calming visual imagery, environmental immersion and narrated soothing metaphors about pain control and deflection. Some patients appreciated the fact that the VR headset blocked sight of doctors and equipment that they found particularly anxiety provoking. Although patients generally reported that the VR did not remove their pain entirely, they reported that the distraction element helped control pain and immediate recovery from instances of sharp pain during the procedure. In contrast, some patients reported no effect of the VR technology on experienced levels of pain or that it was only effective during low to moderate pain. Views were mixed on whether the lack of 
situational awareness of the consultation room was of benefit and some patients preferred to be more aware of the procedure or be able to talk unimpeded with the doctor. A minority of patients reported wearing the VR headset to be uncomfortable and claustrophobic, or that the sense of motion in the VR environment induced nausea.

\section{Discussion}

\section{Main findings}

Compared to standard care, the virtual reality pain management intervention had a large effect in reducing pain and anxiety in outpatient hysteroscopy. This effect was robust, after controlling for baseline pain and anxiety expectations and a range of patient covariates. Staff and majority of the patients found the procedure to be both feasible and acceptable and patients reported a range of experiences, suggestive of the mechanisms by which VR technology may influence pain and anxiety via immersion, relaxation, distraction and imagery.

The study additionally demonstrated willingness of patients to participate and identified barriers to recruitment, non-participation, compliance or standardisation of healthcare providers care pathways through a mixed methods approach using qualitative data to draw useful insights complementing the findings from the quantitative analysis, in order to support future research and development in this area. Insights generated from the themes suggested offering a multimodal pain relief strategy to improve experience at outpatient hysteroscopy. Qualitative analysis suggested patient profiling based on history, taking into consideration patient preferences by offering a variety of distraction techniques with a range of videos to choose from were they to choose virtual reality as a distraction technique. The analysis offered key insights into patient expectations concerning the degree of pain relief possible with virtual reality technology and implementation strategies to facilitate around transfer of research finding into clinical setting.

\section{Strengths and weaknesses}

The topic of pain control in gynaecological procedures is a difficult topic to study and a significant strength of this study lies in the parallel qualitative investigation of patient attitudes and experiences. The experimental arm of this study achieved a $100 \%$ follow-up rate from baseline and was strengthened through the use of standard methods of control, including randomisation, stratification and minimisation techniques ensured comparability at baseline and minimising selection bias. Numeric Rating Scale is known to be a validated measure of pain, is easy to use, has high compliance rates and detects meaningful changes in pain and anxiety $^{25}$.

One limitation of the intervention was that the video was made from a standing rather than prone perspective; the field of vision during hysteroscopy was such that the entire content of the virtual environment could not be explored and this might be addressed by development in the VR technology. Restriction of movement of the patient whilst engaging with the video in light of the nature of the diagnostic procedure could also limit the degree of immersion. The duration of the video was shorter than the length of the procedure for two patients, requiring the video to be restarted. This disrupted the immersion experience and required the health care assistant to keep a watch on when the video finished. Despite these limitations, the intervention was found to be effective in analysis.

The intervention, due to its nature, could not be blinded from the participants, so demand characteristics related to self-reporting of outcome scores may have influenced the results. Non-blinding of the participants could have resulted in patients receiving the VR intervention underreporting the pain and anxiety scores and those patients not receiving the intervention to have over-reported the scores. Additionally, the pain and anxiety scores were measured within 10 minutes of the intervention and were therefore subject to a degree of recall bias. As prior estimates of standard deviation were not available, powering the study for any expected effect size was not possible. However, we detected a relatively large significant difference between groups and therefore avoided the risk of a type 2 error. Our findings will inform sample size calculations for a future full-scale trial.

To our knowledge, this is the first randomised evaluation of feasibility, effectiveness and acceptability of a 
virtual reality intervention in gynaecology. However, a trial protocol has been published for a randomised controlled trial for VR analgesia for women during hysterosalpingograms and results will be forthcoming. ${ }^{34}$

\section{Interpretation of findings}

Ensuring adequate pain relief and allaying anxiety during outpatient hysteroscopy can be challenging and can impact women's satisfaction with the experience. Appropriate patient selection, counselling and adequate pain management during the procedure can improve patient experience, reduce the number of failed procedures, and improve safety, accuracy and effectiveness of the procedure.

There is a lack of consensus on the choice of analgesia for outpatient hysteroscopy ${ }^{9}$ with a recent metaanalysis and systematic review suggesting oral NSAIDS and TENS for pain relief. ${ }^{35}$ Despite this, there has been limited research into the role of distraction techniques in the management of pain and anxiety in ambulatory gynaecological procedures with no published studies on virtual reality as a pain relief modality. ${ }^{26}$ Nonpharmacological options of pain relief at outpatient hysteroscopy include music ${ }^{36,37}$, hypnosis, vaginoscopic methods of hysteroscopy, ${ }^{38}$ adjusting the temperature and pressure of distension medium, stretching of the uterus with a full bladder and electricity via TENS ${ }^{26}$ watching the screen ${ }^{39}$, conversation with positive suggestion and guided imagery. Our study provides new evidence that VR distraction techniques could be used in future to enhance the range of pain relief options.

Our qualitative findings are suggestive of the psychological mechanisms by which VR reduces pain but further research is needed in this area. Interaction with VR uses a substantial amount of the patient's limited controlled attentional resources. ${ }^{40} .{ }^{41}{ }^{42}$. By virtue of spending lesser time thinking about the by via distracting the patients, the intervention may operate to reduce pain scores.

From a service implementation perspective, insights generated from the themes suggested offering a multimodal pain relief strategy to improve experience at outpatient hysteroscopy. Qualitative analysis suggested patient profiling based on history, taking into consideration patient preferences by offering a variety of distraction techniques with a range of videos to choose from were they to choose virtual reality as a distraction technique. The analysis offered key insights around managing patient expectations around the degree of pain relief with virtual reality and implementation strategies around transferring research finding into clinical setting.

The study showed a large sized reduction in scores in pain or anxiety with virtual reality, even though it is unlikely to eliminate pain completely. The intervention was well tolerated with no serious side effects. It would be useful to compile core outcome sets based on patient reported outcomes for pain and anxiety towards future research in ambulatory gynaecological procedures. Algorithmic prediction of the types of patients who would benefit most from the intervention should also be modelled in future trials based on patient characteristics and baseline pain and anxiety scores.

The type of VR equipment and the degree of interaction with the video is likely to effect the analgesic effectiveness. ${ }^{19}$ Virtual reality is an evolving technology and designing appropriate content of the video with adequate duration, headsets and hygiene masks to comply with infection control protocols and also have affordances and good aesthetics that make it comfortable to wear would be paramount prior to clinical adoption, which would need codesign with patients and manufacturers. It would be appropriate to have a range of videos for the patient to choose from, which might be with our without narration. Other avenues include using virtual reality for patient education for familiarisation with the procedure and using it as a triage prior to offering it as an intervention for pain relief.

\section{Conclusions}

Immersive virtual reality intervention is feasible, effective and acceptable in a clinical setting as a distraction technique for the management of pain and anxiety in patients undergoing outpatient hysteroscopy. This study demonstrated a robust effect for VR technology in this application, within a relatively small-scale trial. Future development of VR technologies for this application, coupled with larger-scale trials, would strengthen the evidence-base for alternative pain management interventions in ambulatory gynaecology. 
Transferability of these findings into the clinical setting needs to be evaluated by future trials and economic evaluations of additional costs of equipment and training.

Disclosure of Interests : None to declare

\section{Contribution to authorship:}

The study was a part of a Dissertation for ND, for an MSc In Health Care and Design, Imperial College London. ND was involved in the writing of the drafts of the study protocol and manuscripts, data collection and was the outcome assessor. JB was the study supervisor and was involved in the writing of the drafts of the study protocol and manuscript, reviewing the draft of the statistical analysis plans and interpretation of results. KSK was involved in the study set up and writing of the draft of the manuscript and interpretation of results. JM was involved in collation of the data. JA was involved in the set up of the study and randomisation and reviewed the draft of the manuscript. FJGC was involved in the final data analysis. GF was a study supervisor and involved in the study design and write up of the study protocol.

Data analysis, interpretation of findings and writing the manuscript was supported by funding from the National Institute of Health Research (NIHR) Yorkshire and Humber Patient Safety Translational Research Centre. The views expressed are those of the authors, and not necessarily those of the NIHR or the Department of Health and Social Care.

Details of Ethics approval: Newcastle and North Tyneside1 National Research Ethics Committee. United Kingdom. Approval Date- 29/5/2018. Reference 18/NE/0165.

Data Access and Responsibility: The principal investigator, Nandita Deo, had full access to all of the data in the study and takes responsibility for the integrity of the data and the accuracy of the data analysis.

Funding: Supported by NIHR Patient safety Translational Research Centre funding.

\section{Acknowledgements:}

We thank all the women who consented to participate in the trial as well as all nurses and gynaecologists who performed the procedure: their enthusiasm and support as co-researchers in this clinical project was indispensable. A special thanks for Jayshree Patel and Chris Astley, the lovely couple who helped codesign the study and gave a patient's perspective along with technical expertise. We are grateful to Dr Sheila Popert, Medical Director at St Giles Hospice for commissioning the video and requesting David Attenborough to lend his voice for the narration in the Video, Forest of Serenity that was the centre stone for the intervention. We are indebted to Anna Wojdecka, Visiting Lecturer, Healthcare \& Design at the Royal College of Art, London, for providing design ideas and academic rigor in the conduct of this study.

Tables and Figures

Figure 1: Patient recruitment in the trial.

Table 1. Baseline characteristics of participants allocated to standard care or virtual reality intervention in the Trial

\begin{tabular}{|c|c|c|c|c|}
\hline Characteristic & $\begin{array}{l}\text { Standard care (n } \\
=20) \text { Mean (SD) } \\
\text { or } n(\%)\end{array}$ & $\begin{array}{l}\text { Standard care (n } \\
=20) \text { Mean }(S D) \\
\text { or } n(\%)\end{array}$ & $\begin{array}{l}\text { Virtual reality (n } \\
=20) \text { Mean (SD) } \\
\text { or } n(\%)\end{array}$ & $\begin{array}{l}\text { Virtual reality ( } \mathrm{n} \\
=20 \text { ) Mean (SD) } \\
\text { or } n(\%)\end{array}$ \\
\hline Age (years) & Age (years) & $31.3(5.2)$ & $31.3(5.2)$ & $31.1(5.4)$ \\
\hline Parity (No.) & Parity (No.) & $2.2(1.9)$ & $2.2(1.9)$ & $2.4(1.7)$ \\
\hline Nulliparous & Nulliparous & $4(20)$ & $4(20)$ & $4(20)$ \\
\hline Multiparous & Multiparous & $16(80)$ & $16(80)$ & $16(80)$ \\
\hline Ethnicity & Ethnicity & & & \\
\hline White & White & $8(40)$ & $8(40)$ & $9(45)$ \\
\hline Black & Black & $4(20)$ & $4(20)$ & $3(15)$ \\
\hline
\end{tabular}




\begin{tabular}{|c|c|c|c|c|}
\hline Characteristic & $\begin{array}{l}\text { Standard care (n } \\
=20) \text { Mean }(\mathrm{SD}) \\
\text { or } \mathrm{n}(\%)\end{array}$ & $\begin{array}{l}\text { Standard care (n } \\
=20) \text { Mean }(\mathrm{SD}) \\
\text { or } \mathrm{n}(\%)\end{array}$ & $\begin{array}{l}\text { Virtual reality ( } \mathrm{n} \\
=20) \text { Mean (SD) } \\
\text { or } n(\%)\end{array}$ & $\begin{array}{l}\text { Virtual reality ( } \mathrm{n} \\
=20) \text { Mean }(\mathrm{SD}) \\
\text { or } n(\%)\end{array}$ \\
\hline Asian & Asian & $5(25)$ & $5(25)$ & $8(40)$ \\
\hline Mixed & Mixed & $3(15)$ & $3(15)$ & $0(0)$ \\
\hline $\begin{array}{l}\text { Menopausal } \\
\text { status }\end{array}$ & $\begin{array}{l}\text { Menopausal } \\
\text { status }\end{array}$ & & & \\
\hline $\begin{array}{l}\text { Pre- } \\
\text { menopausal }\end{array}$ & $\begin{array}{l}\text { Pre- } \\
\text { menopausal }\end{array}$ & $7(35)$ & $7(35)$ & $7(35)$ \\
\hline $\begin{array}{l}\text { Post- } \\
\text { menopausal }\end{array}$ & $\begin{array}{l}\text { Post- } \\
\text { menopausal }\end{array}$ & $13(65)$ & $13(65)$ & $13(65)$ \\
\hline $\begin{array}{l}\text { Prior } \\
\text { outpatient } \\
\text { hysteroscopy }\end{array}$ & $\begin{array}{l}\text { Prior } \\
\text { outpatient } \\
\text { hysteroscopy }\end{array}$ & $3(15)$ & $3(15)$ & $4(20)$ \\
\hline Hysteroscopy & Hysteroscopy & & & \\
\hline indication & indication & & & \\
\hline $\begin{array}{l}\text { Heavy Menstrual } \\
\text { Bleeding }\end{array}$ & $\begin{array}{l}\text { Heavy Menstrual } \\
\text { Bleeding }\end{array}$ & $5(25)$ & $5(25)$ & $6(30)$ \\
\hline Incidental finding & Incidental finding & $2(10)$ & $2(10)$ & $5(25)$ \\
\hline $\begin{array}{l}\text { Postmenopausal } \\
\text { bleeding }\end{array}$ & $\begin{array}{l}\text { Postmenopausal } \\
\text { bleeding }\end{array}$ & $11(55)$ & $11(55)$ & $8(40)$ \\
\hline Lost coil thread & Lost coil thread & $2(10)$ & $2(10)$ & $0(0)$ \\
\hline $\begin{array}{l}\text { Recurrent } \\
\text { postcoital } \\
\text { bleeding }\end{array}$ & $\begin{array}{l}\text { Recurrent } \\
\text { postcoital } \\
\text { bleeding }\end{array}$ & $0(0)$ & $0(0)$ & $1(5)$ \\
\hline $\begin{array}{l}\text { Pain killers } \\
\text { taken before } \\
\text { procedure }\end{array}$ & $\begin{array}{l}\text { Pain killers } \\
\text { taken before } \\
\text { procedure }\end{array}$ & $12(60)$ & $12(60)$ & $13(65)$ \\
\hline $\begin{array}{l}\text { Expected pain } \\
\text { score }\end{array}$ & $\begin{array}{l}\text { Expected pain } \\
\text { score }\end{array}$ & $6.5(2.0)$ & $6.5(2.0)$ & $7.0(2.2)$ \\
\hline $\begin{array}{l}\text { Expected } \\
\text { anxiety score }\end{array}$ & $\begin{array}{l}\text { Expected } \\
\text { anxiety score }\end{array}$ & $5.6(3.1)$ & $5.6(3.1)$ & $6.4(2.9)$ \\
\hline
\end{tabular}

Table 2. Comparison of experienced pain and anxiety between standard care and virtual reality intervention in the Trial

\begin{tabular}{lllll}
\hline Group & Group & $\mathbf{n}$ & Mean (SD) & $\mathbf{9 5 \%}$ Confidence Interva \\
\hline & Worst Pain Scores & Worst Pain Scores & Worst Pain Scores & Worst Pain Scores \\
Standard Care & Standard Care & 20 & $7.85(2.56)$ & 6.65 \\
Virtual Reality & Virtual Reality & 20 & $5.65(2.41)$ & 4.52 \\
Difference & Difference & & 2.2 & 3.79 \\
& Average Pain Scores & Average Pain Scores & Average Pain Scores & Average Pain Scores \\
Standard Care & Standard Care & 20 & $6(2.62)$ & 4.78 \\
Virtual Reality & Virtual Reality & 20 & $3.7(2.66)$ & 2.46 \\
Difference & Difference & & 2.3 & 0.61 \\
& Anxiety Scores & Anxiety Scores & Anxiety Scores & Anxiety Scores \\
Standard Care & Standard Care & 20 & $5.45(3.35)$ & 3.88 \\
Virtual Reality & Virtual Reality & 20 & $3.3(2.03)$ & 2.35 \\
Difference & Difference & & 2.15 & 0.38 \\
\hline
\end{tabular}


Table 3. Multiple regression results for effect of virtual reality condition on experienced worst pain, average pain and anxiety scores, after adjusting parity, menopausal condition and cervical stenosis

\begin{tabular}{lll}
\hline Model & Model & Model R2 \\
\hline Worst Pain Scores & Worst Pain Scores & 0.24 \\
Average Pain Scores & Average Pain Scores & 0.24 \\
Anxiety Scores & Anxiety Scores & 0.16 \\
\hline
\end{tabular}

Appendix S1: Multiple regression analysis of clinical outcomes in the trial: Full model parameters

Model for worst pain scores

\begin{tabular}{|c|c|c|c|c|c|}
\hline & $\begin{array}{l}\text { term } \\
\langle\text { chr }\rangle\end{array}$ & $\begin{array}{r}\text { estimate } \\
\langle\mathrm{dbl}\rangle\end{array}$ & $\begin{array}{r}\text { std.error } \\
\langle d b l\rangle\end{array}$ & $\begin{array}{r}\text { statistic } \\
\langle\mathrm{dbl}\rangle\end{array}$ & $\begin{array}{r}\text { p.value } \\
\langle d b l\rangle\end{array}$ \\
\hline 1 & (Intercept) & 8.22 & 0.912 & 9.02 & 0 \\
\hline 2 & treatVR & -2.11 & 0.788 & -2.67 & 0.0113 \\
\hline 3 & parity & -0.0122 & 0.225 & -0.0541 & 0.957 \\
\hline 4 & endo_c_stenTRUE & 1.79 & 1.1 & 1.63 & 0.112 \\
\hline 5 & menoppostmenopausal & -1.09 & 0.874 & -1.24 & 0.222 \\
\hline
\end{tabular}

Model for average pain scores

$\begin{array}{lrrrr}\text { term } & \text { estimate } & \text { std.error } & \text { statistic } & \text { p.value } \\ \text { 〈chr } & \langle\text { dbl } & \langle\text { dbl } & \langle\text { dbl } & \langle\text { dbl } \\ 1 \text { (Intercept) } & 5.69 & 0.966 & 5.89 & 0 \\ 2 \text { treatVR } & -2.28 & 0.835 & -2.74 & 0.0097 \\ 3 \text { parity } & 0.26 & 0.239 & 1.09 & 0.283 \\ 4 \text { endo_c_stenTRUE } & 1.61 & 1.16 & 1.38 & 0.175 \\ 5 \text { menoppostmenopausal } & -0.88 & 0.926 & -0.951 & 0.348\end{array}$

Model for anxiety scores

\begin{tabular}{|c|c|c|c|c|c|}
\hline & $\begin{array}{l}\text { term } \\
\langle\text { chr }\rangle\end{array}$ & $\begin{array}{r}\text { estimate } \\
\langle d b l\rangle\end{array}$ & $\begin{array}{r}\text { std.error } \\
\langle d b l\rangle\end{array}$ & $\begin{array}{r}\text { statistic } \\
\langle d \mathrm{dbl}\rangle\end{array}$ & $\begin{array}{r}\text { p. value } \\
\langle\text { dbl }\rangle\end{array}$ \\
\hline 1 & (Intercept) & 4.97 & 1.04 & 4.76 & 0 \\
\hline 2 & treatVR & -2.13 & 0.902 & -2.36 & 0.0241 \\
\hline 3 & parity & 0.133 & 0.258 & 0.516 & 0.609 \\
\hline 4 & endo_c_stentRUE & 1.14 & 1.26 & 0.904 & 0.372 \\
\hline 5 & menoppostmenopausal & -0.0571 & 1.00 & -0.0571 & 0.955 \\
\hline
\end{tabular}

Appendix S2: Patients' experiences of undergoing the VR distraction technique: Representative perspectives based upon example quotations from qualitative data

Positive experiences:

- I think it was really beautiful; it made me feel more relaxed and made me feel, not think so much of the pain. But it was really lovely to watch, very peaceful. 
- That's very good, when you're watching that you feel like you're moving with it. But it is very, very good. It is worth having, I mean when someone's in pain, it's hard to take your mind off. But it's soothing, it's relaxing. Because of the scenery around and the way it's going.

- The VR goggles helped me. My concentration, when it talked about the marble, putting all your pain in the marble and throwing it.

- I quite liked the idea when the narrator talked about the pain, because it is painful, you can't ignore it. Having someone tell you it's going to go helps you believe that it's going to pass.

- Very colourful, background was calm, very different plants and just the things he was saying took my mind off the pain.

- I feel the VR is really good. I feel like you're not thinking about what's happening next in the procedure. I feel like it just takes your mind off of what's going on. I feel like it's very helpful and it's a good distraction. It should be used.

- In future, if you apply this method instead of putting patients under General Anaesthetic or Local Anaesthetic, those take more time and recovery. At the beginning it was a bit uncomfortable, but then I got used to it.

- When I see doctors around me, I am more anxious. I am more anxious when people are walking around. I prefer not to see this around me. I prefer not to see instruments around me. The VR enabled this to happen.

- I felt like I was in the forest. I thought that you hadn't started the procedure at all.

- Sharp pain lasted 5-6 seconds couple of times. That's when I lost focus from the video. But then it does help as it says your pain's going to ebb away and it does sort of go away. It seemed to work for me.

- Don't really know. What I was watching, that took my mind off of it a lot. It was helping. It helped that I was watching that rather than watching the screen or the doctor.

- It was really good. Took my mind off of it, and it helped a lot. At first I didn't think I would be able to keep them on, because I was so anxious. But I'm really glad that I did.

- Those sharp pains nothing could have helped I think, but generally, just being relaxed and having that distraction. Maybe if I hadn't had that and I was just looking at you guys and the equipment, my anxiety and pain would have been worse.

\section{Negative experiences:}

- I declined to use the headset, as I felt uncomfortable and claustrophobic.

- I initially said yes to the intervention; but I did not know what to expect. I thought that it would be like normal glasses and though that I could see a movie through this. But these glasses pressed around my face and my nose was pressed and I couldn't breathe and it was uncomfortable and I felt claustrophobic.

- People definitely need something, I'm glad I took some painkillers before

- It was a good idea, because it was quite interesting to watch, but during the middle I just shut my eyes anyways so I wasn't watching anything.

- It did at the beginning, it was interesting to have a look around, but it didn't help with the actual really bad pain.

- It was good. But it made you feel a bit sick when it moved. Like when the picture moves deeper into the forest. Those made me feel a bit sick. Didn't like that the moving. That was the point I wanted to take the glasses off but I kept it on.

- I am claustrophobic. I like a movie, but that might suit a lot of people. Because of the calming voice. He has got a calming voice, once you get started you can move about and look around. But that may help a lot of people. But I'd rather know what's going on. Prefer to watch screen.

\section{References}

1. RCOG Press. RCOG. Standards for gynaecology. 2008. 
2. Finikiotis G. Outpatient hysteroscopy: pain assessment by visual analogue scale. Aust N Z J Obstet Gynaecol. 1990 Feb; 30(1): 89-90.

3. Cooper NAM, Khan KS, Clark TJ. Local anaesthesia for pain control during outpatient hysteroscopy: systematic review and meta-analysis. BMJ. 2010 Mar 23;340:c1130.

4. Zupi E, Luciano AA, Valli E, Marconi D, Maneschi F, Romanini C. The use of topical anesthesia in diagnostic hysteroscopy and endometrial biopsy**Presented at the 22nd Annual Meeting of the American Association of Gynecologic Laparoscopists, San Francisco, California, November 10 to 14, 1993. Fertility and Sterility. 1995 Feb 1;63(2):414-6.

5. Shahid A, Pathak M, Gulumser C, Parker S, Palmer E, Saridogan E. Optimum uterine filling pressure for outpatient diagnostic hysteroscopy: a double-blind, randomized controlled trial. Reproductive BioMedicine Online. 2014 Jan;28(1):86-91.

6. de Carvalho Schettini JA, Ramos de Amorim MM, Ribeiro Costa AA, Albuquerque Neto LC. Pain evaluation in outpatients undergoing diagnostic anesthesia-free hysteroscopy in a teaching hospital: a cohort study. J Minim Invasive Gynecol. 2007 Nov;14(6):729-35.

7. Cicinelli E. Hysteroscopy without Anesthesia: Review of Recent Literature. Journal of Minimally Invasive Gynecology. 2010 Nov;17(6):703-8.

8. Ahmad G, Saluja S, O'Flynn H, Sorrentino A, Leach D, Watson A. Pain relief for outpatient hysteroscopy. Cochrane Database of Systematic Reviews [Internet]. 2017 [cited 2018 Oct 30];(10). Available from: https://www.cochranelibrary.com/cdsr/doi/10.1002/14651858.CD007710.pub3/abstract

9. O'Flynn H, Murphy LL, Ahmad G, Watson AJ. Pain relief in outpatient hysteroscopy: a survey of current UK clinical practice. Eur J Obstet Gynecol Reprod Biol. 2011 Jan;154(1):9-15.

10. Allen RH, Micks E, Edelman A. Pain relief for obstetric and gynecologic ambulatory procedures. Obstetrics and Gynecology Clinics of North America. 2013 Dec;40(4):625-45.

11. Calvo JS, Del Valle Rubido C. Review of analgesic and anesthetic methods for office hysteroscopy. Gynecological Surgery. 2016;13 (1 Supplement 1):S402.

12. del Valle C, Solano JA, Rodríguez A, Alonso M. Pain management in outpatient hysteroscopy. Gynecology and Minimally Invasive Therapy. 2016 Nov 1;5(4):141-7.

13. Loreto-Quijada D, Gutierrez-Maldonado J, Nieto R, Gutierrez-Martinez O, Ferrer-Garcia M, Saldana C, et al. Differential effects of two virtual reality interventions: distraction versus pain control. Cyberpsychol Behav Soc Netw. 2014 Jun;17(6):353-8.

14. Chan E, Foster S, Sambell R, Leong P. Clinical efficacy of virtual reality for acute procedural pain management: A systematic review and meta-analysis. PLOS ONE. 2018 Jul 27;13(7):e0200987.

15. Dascal J, Reid M, IsHak WW, Spiegel B, Recacho J, Rosen B, et al. Virtual Reality and Medical Inpatients: A Systematic Review of Randomized, Controlled Trials. Innov Clin Neurosci. 2017 Feb;14(12):14-21.

16. Garrett B, Taverner T, Masinde W, Gromala D, Shaw C, Negraeff M. A rapid evidence assessment of immersive virtual reality as an adjunct therapy in acute pain management in clinical practice. Clin J Pain. 2014 Dec;30(12):1089-98.

17. Gold JI, Belmont KA, Thomas DA. The Neurobiology of Virtual Reality Pain Attenuation. CyberPsychology \& Behavior. 2007 Aug 1;10(4):536-44.

18. Hoffman HG. Virtual-reality therapy. Sci Am. 2004 Aug;291(2):58-65. 
19. Hoffman HG, Chambers GT, Meyer WJ 3rd, Arceneaux LL, Russell WJ, Seibel EJ, et al. Virtual reality as an adjunctive non-pharmacologic analgesic for acute burn pain during medical procedures. Ann Behav Med. 2011 Apr;41(2):183-91.

20. Hoffman HG, Garcia-Palacios A, Thomas AK, Schmidt A. Virtual reality monitoring: phenomenal characteristics of real, virtual, and false memories. Cyberpsychol Behav. 2001 Oct;4(5):565-72.

21. Li A, Montaño Z, Chen VJ, Gold JI. Virtual reality and pain management: current trends and future directions. Pain Management. 2011 Mar 1;1(2):147-57.

22. Raghav K, Van Wijk AJ, Abdullah F, Islam MN, Bernatchez M, De Jongh A. Efficacy of virtual reality exposure therapy for treatment of dental phobia: a randomized control trial. BMC Oral Health. 2016 Feb $27 ; 16: 25$.

23. Freeman D, Haselton P, Freeman J, Spanlang B, Kishore S, Albery E, et al. Automated psychological therapy using immersive virtual reality for treatment of fear of heights: a single-blind, parallel-group, randomised controlled trial. The Lancet Psychiatry. 2018 Aug 1;5(8):625-32.

24. Powers MB, Emmelkamp PMG. Virtual reality exposure therapy for anxiety disorders: A meta-analysis. Journal of Anxiety Disorders. 2008 Apr 1;22(3):561-9.

25. Frey DP, Bauer ME, Bell CL, Low LK, Hassett AL, Cassidy RB, et al. Virtual Reality Analgesia in Labor: The VRAIL Pilot Study-A Preliminary Randomized Controlled Trial Suggesting Benefit of Immersive Virtual Reality Analgesia in Unmedicated Laboring Women. Anesth Analg. 2018 Jul 11;

26. Amer-Cuenca JJ, Marín-Buck A, Vitale SG, Rosa VLL, Caruso S, Cianci A, et al. Non-pharmacological pain control in outpatient hysteroscopies. Minimally Invasive Therapy \& Allied Technologies. 2020 Jan $2 ; 29(1): 10-9$.

27. Forest Of Serenity [Internet]. St Giles Hospice. [cited 2018 Nov 22]. Available from: https://www.stgileshospice.com/forest-of-serenity/

28. Breivik H, Borchgrevink PC, Allen SM, Rosseland LA, Romundstad L, Breivik Hals EK, et al. Assessment of pain. Br J Anaesth. 2008 Jul 1;101(1):17-24.

29. Breivik H, Borchgrevink PC, Allen SM, Rosseland LA, Romundstad L, Breivik Hals EK, et al. Assessment of pain. Br J Anaesth. 2008 Jul 1;101(1):17-24.

30. Kindler CH, Harms C, Amsler F, Ihde-Scholl T, Scheidegger D. The visual analog scale allows effective measurement of preoperative anxiety and detection of patients' anesthetic concerns. Anesth Analg. 2000 Mar;90(3):706-12.

31. Dearnley C. A reflection on the use of semi-structured interviews. Nurse Res. 2005;13(1):19-28.

32. Pope C, Ziebland S, Mays N. Analysing qualitative data. BMJ. 2000 Jan 8;320(7227):114-6.

33. Cohen J. Statistical Power Analysis for the Behavioral Sciences [Internet]. Elsevier; 1977 [cited 2019 Dec 19]. Available from: https://linkinghub.elsevier.com/retrieve/pii/C2013010517X

34. Wang Y-L, Gao H-X, Wang J-S, Wang J-H, Bo L, Zhang T-T, et al. Immersive virtual reality as analgesia for women during hysterosalpingography: study protocol for a randomized controlled trial. Trials [Internet]. 2020 Jan 20 [cited 2020 Feb 21];21. Available from: https://www.ncbi.nlm.nih.gov/pmc/articles/PMC6972014/

35. De Silva PM, Mahmud A, Smith PP, Clark TJ. Analgesia for office hysteroscopy: systematic review \& meta-analysis. Journal of Minimally Invasive Gynecology [Internet]. 2020 Jan 23 [cited 2020 Feb 21]; Available from: http://www.sciencedirect.com/science/article/pii/S1553465020300467 
36. Angioli R, Nardone CDC, Plotti F, Cafà EV, Dugo N, Damiani P, et al. Use of Music to Reduce Anxiety during Office Hysteroscopy: Prospective Randomized Trial. Journal of Minimally Invasive Gynecology. 2014 May 1;21(3):454-9.

37. Mak N, Reinders IMA, Slockers SA, Westen E, Maas JWM, Bongers MY. The effect of music in gynaecological office procedures on pain, anxiety and satisfaction: a randomized controlled trial. Gynecol Surg. 2017;14(1):14.

38. Cooper NA, Smith P, Khan KS, Clark TJ. Vaginoscopic approach to outpatient hysteroscopy: a systematic review of the effect on pain [Internet]. Centre for Reviews and Dissemination (UK); 2010 [cited 2020 Feb 21]. Available from: https://www.ncbi.nlm.nih.gov/books/NBK80588/

39. Ogden J, Heinrich M, Potter C, Kent A, Jones S. The impact of viewing a hysteroscopy on a screen on the patient's experience: a randomised trial. BJOG: An International Journal of Obstetrics \& Gynaecology. 2009 Jan 1;116(2):286-93.

40. Kahneman D. Attention and effort. Englewood Cliff, NJ: Prentice Hall; 1973.

41. Eccleston C. Role of psychology in pain management. British Journal of Anaesthesia. 2001 Jul $1 ; 87(1): 144-52$.

42. Eccleston C, Crombez G. Pain demands attention: A cognitive-affective model of the interruptive function of pain. Psychological Bulletin. 1999;125(3):356-66.

\section{Hosted file}

BJOG figures.docx available at https://authorea.com/users/303133/articles/433269-virtualreality-for-acute-pain-in-outpatient-hysteroscopy-a-randomised-controlled-trial

\section{Hosted file}

BJOG Tables and Figures.docx available at https://authorea.com/users/303133/articles/433269virtual-reality-for-acute-pain-in-outpatient-hysteroscopy-a-randomised-controlled-trial 